\title{
Lumbar Sympathetic Block for Bilateral Post-Prostatectomy Lower Extremity Pain in the Femoral Nerve Distribution
}

\author{
Fadi Farah", Antranig Kalaydjian, Yuen Cheng, Pascal Scemama and Martin Acquadro
}

Department of Anesthesiology and Pain Medicine, St Elizabeth Medical Center, USA

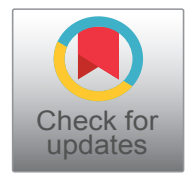

*Corresponding author: Fadi Farah, Department of Anesthesiology and Pain Medicine, St Elizabeth Medical Center, Boston, MA, USA, E-mail: fhff@hotmail.com

\begin{abstract}
Objective: Lower extremities nerves damage is a known complication of prostatectomies. Lumbar sympathetic block is a well-established treatment for sympathetically-mediated lower extremity pain. We report a case of bilateral lower extremity pain in a femoral distribution that developed after a robotic assisted prostatectomy and resolved after a lumbar sympathetic block.

Case Report: A 69-year-old male patient presented with bilateral thigh pain one month after an uneventful robotic-assisted laparoscopic prostatectomy in the femoral nerve distribution. CT scan was unremarkable save for expected postsurgical changes. The patient failed conservative treatment. Considering a possible sympathetically-mediated pain, we performed a right lumbar sympathetic block that improved his pain.

Conclusions: A lumbar sympathetic block can be used a salvage therapy when conservative management fails.
\end{abstract}

\section{Introduction}

Robotic assisted laparoscopic radical prostatectomy is a treatment for organ confined prostate cancer [1]. Lower extremities nerves damage is a known complication of prostatectomies [2]. The rate of this complication is estimated to be $1.3-1.7 \%[1,2]$. Femoral nerve neuropathy is described in open prostatectomies [3] and robotic-assisted laparoscopic prostatectomies [1]. Most of these neuropathies result in mild self-limited peripheral nerve injuries, with resolution usually within a few weeks. However, few patients develop prolonged severe debilitating pain up to a year. We report a case of bilateral lower extremity pain in a femoral distribution which developed after a robotic assisted prostatectomy and responded favorably to lumbar sympathetic block.

\section{Materials and Methods}

Patient informed consent was obtained for submission of the case report.

\section{Case Presentation}

A 69-year-old male patient with a history of well controlled noninsulin-dependent diabetes presented to the pain clinic with unbearable bilateral thigh pain. One month prior to presentation he underwent an uncomplicated robotic-assisted laparoscopic nerve sparing radical prostatectomy with lymph node dissection. Three days after an uneventful postoperative course, he developed a worsening sharp, shooting pain involving the L1, L2, L3, L4 dermatomes, worse on the right side, exacerbated by weather changes, associated with allodynia and hyperalgesia over the affected areas. There was no associated motor weakness, swelling, fever, or muscle spasm. CT scan of the abdomen and pelvis with and without contrast were unremarkable save for expected postsurgical changes.

The patient failed conservative treatment including Amitriptyline, Gabapentin, Zonisamide, Duloxetine, and Oxycodone. Due to lack of response to neuropathic medications and a possibly sympathetically-mediated pain, we attempted a right lumbar sympathetic block the mid $L 3$ vertebral body. On follow-up, the patient reported $80 \%$ pain reduction, with pain reported as $2 / 10$ which decreased from $8-9 / 10$ pre-injection and an increase in function.

\section{Discussion}

Femoral nerve injuries in laparoscopic prostatecto-

Citation: Farah F, Kalaydjian A, Cheng Y, Scemama P, Acquadro M (2018) Lumbar Sympathetic Block for Bilateral Post-Prostatectomy Lower Extremity Pain in the Femoral Nerve Distribution. Int J Anesthetic Anesthesiol 5:070. doi.org/10.23937/2377-4630/1410070

Accepted: July 24, 2018: Published: July 26, 2018

Copyright: (c) 2018 Farah F, et al. This is an open-access article distributed under the terms of the Creative Commons Attribution License, which permits unrestricted use, distribution, and reproduction in any medium, provided the original author and source are credited. 
mies are reported extensively in the Literature [4]. Risk factors for femoral nerve injury include increased surgical time, split-leg positioning, Book walter or self-retaining retractor use, compression of the psoas muscle or the lateral pelvic wall, high BMI, variant anatomy, diabetes, and peripheral vascular disease [4-6]. In the lithotomy position, the hip is flexed, abducted, and externally rotated leading the femoral nerve to be sharply angulated beneath the inguinal ligament, putting it at risk [4]. Warner and associates estimated that for each hour in the lithotomy position, the risk for motor neuropathy increases 100 -fold [7]. This patient has diabetes, a normal BMI, and underwent a surgery of less than three hours duration in lithotomy position, without use of retractors or muscle/pelvic wall compression. The bilateral presentation of the pain several days after the procedure makes direct surgical injury to the femoral nerve unlikely. The $\mathrm{CT}$ abdomen and pelvis done postoperatively did not show any hematoma or lymphocele. The presentation of pain and allodynia out of proportion to examination, affected by weather changes without sensory or motor deficit suggests that the pain was sympathetically mediated; hence the lack of response to neuropathic medications. The traditional treatment for femoral neuropathy include physical therapy, avoidance of excessive hip abduction and external rotation, and knee bracing to prevent buckling of the knee. Neuropathic pain have been used with variable success.

The lumbar sympathetic trunk is situated on the anterolateral aspect of lumbar vertebral bodies and courses medially to the psoas muscle, exiting over the sacral ala towards the pelvis [8]. Anatomic variations of the ganglia located adjacent to the iliac vessels have been reported [9]. This surgery involved Para-iliac lymph node resection. Lumbar sympathetic block is a well-es- tablished treatment for sympathetically-mediated lower extremity pain [8]. We suspected that this patient's pain was related to compression or traumatic tissue damage involving prostatic visceral afferents and autonomic innervation or an anatomic variant of the lumbar sympathetic ganglia.

\section{References}

1. Koç G, Tazeh NN, Joudi FN, Winfield HN, Tracy CR, et al. (2012) Lower extremity neuropathies after robot-assisted laparoscopic prostatectomy on a split-leg table. J Endourol 26: 1026-1029.

2. Manny TB, Gorbachinsky I, Hemal AK (2010) Lower extremity neuropathy after robot assisted laparoscopic radical prostatectomy and radical cystectomy. Can J Urol 17: 5390-5393.

3. Katirji MB, Lanska DJ (1990) Femoral mononeuropathy after radical prostatectomy. Urology 36: 539-540.

4. Mills JT, Burris MB, Warburton DJ, Conaway MR, Schenkman NS, et al. (2013) Positioning injuries associated with robotic assisted urological surgery. J Urol 190: 580-584.

5. Bohrer JC, Walters MD, Park A, Polston D, Barber MD (2009) Pelvic nerve injury following gynecologic surgery: A prospective cohort study. Am J Obstet Gynecol 201: 531-537.

6. Inoue T, Nishikawa K, Takazawa T, Saito S, Goto F (2008) Cases of femoral nerve palsy after radical prostatectomy. Masui 57: 363-365.

7. Warner MA, Martin JT, Schroeder DR, Offord KP, Chute CG (1994) Lower-extremity motor neuropathy associated with surgery performed on patients in a lithotomy position. Anesthesiology 81: 6-12.

8. Beveridge TS, Fournier DE, Groh AMR, Johnson M, Power NE, et al. (2018) The anatomy of the infrarenal lumbar splanchnic nerves in human cadavers: Implications for retroperitoneal nerve-sparing surgery. J Anat 232: 124-133.

9. Gunduz $\mathrm{OH}$, Kenis-Coskun $\mathrm{O}$ (2017) Ganglion blocks as a treatment of pain: Current perspectives. J Pain Res 10: 2815-2826. 